\title{
Survey of Parental Use of Antimicrobial Drugs for Common Childhood Infections, China
}

\author{
Leesa Lin, Stephan Harbarth, Xiaomin Wang, Xudong Zhou
}

In a large-scale survey of 9,526 parents in China, we investigated antimicrobial drug use for common childhood infections. Of children with self-limiting conditions, formal care was sought for $69.2 \%$; of those, $53.4 \%$ received drug prescriptions, including $11.2 \%$ from parental demands. Where drugs were taken without prescriptions, $70 \%$ were from community pharmacies.

ح hina accounts for half of global consumption of antimicrobial drugs $(1,2)$ and reports high rates of antimicrobial resistance, especially among children $(3,4)$. Despite current regulations requiring a prescription to obtain antibiotics, unsupervised use in China is pervasive $(3,4)$. To date, the few studies available on inappropriate administration to children beyond clinical settings have been limited in scope to smallscale data in one geographic area $(3,5-7)$. To inform control strategies, we investigated the prevalence of inappropriate antibiotic use for common childhood infections by parents across different geographic areas and economic development stages, overall parental knowledge about antimicrobial drug use and resistance, the impact of parental pressure for antibiotics on prescribing behaviors, and parental contribution to the overall use of antibiotics in children.

\section{The Study}

Study methods, including sampling strategy and data collection, were previously reported (8). Our data came from a cross-sectional survey conducted during June 2017-April 2018, which recruited 9,526 parents with children $0-13$ years of age across 3 provinces (Zhejiang, Shaanxi, and Guangxi) representing different geographic areas and economic development

Author affiliations: London School of Hygiene and Tropical

Medicine, London, UK (L. Lin); University of Geneva Hospitals and Faculty of Medicine, Geneva, Switzerland (S. Harbarth); Zhejiang University, Hangzhou, China (X. Wang, X. Zhou)

DOI: https://doi.org/10.3201/eid2607.190631 stages in China (9). We conducted multistage stratified random cluster sampling in provinces, prefecture-level cities, urban and rural areas, and local sampling sites that included primary schools (children 6-13 years of age), kindergartens (3-5 years), and community-based health centers ( $0-2$ years). The Institutional Review Board at the Zhejiang University School of Medicine (approval no. ZGL201706-2) and London School of Hygiene and Tropical Medicine (approval no. 14678) reviewed and exempted the study protocol and survey.

We recruited parents through their children, and all participants gave informed consent. We distributed a questionnaire to parents who self-identified as the primary caregiver and healthcare decision maker for the children; parents completed the questionnaire electronically. We collected data on parental sociodemographics, knowledge about antibiotic use and resistance, and last episode of illnesses and treatments experienced by the child within a month (Figure 1).

We calculated an $89 \%$ response rate by dividing the number of parents who completed the survey by the number of parents who were invited to take the survey $(13,680 / 15,424)$. For data quality control, we used measures (including trap questions and IP address control) to detect random responses or duplications, resulting in $70 \%(9,526 / 13,680)$ of the completed surveys verified as valid. We classified missing data $(<11 \%)$ as missing-completely-at-random and excluded from the final analysis.

We conducted descriptive analysis on the data, followed by unadjusted univariable analysis to examine the association between antibiotic prescriptions and parental demand and employed multivariable logistic regression and likelihood ratio tests for adjusted analyses, controlling for relevant sociodemographic variables. We observed profound regional differences in parental socioeconomic composition, antibiotic-use practices, and medical facilities used when children (age 5.8 years, SD \pm 3.6 years) were ill 


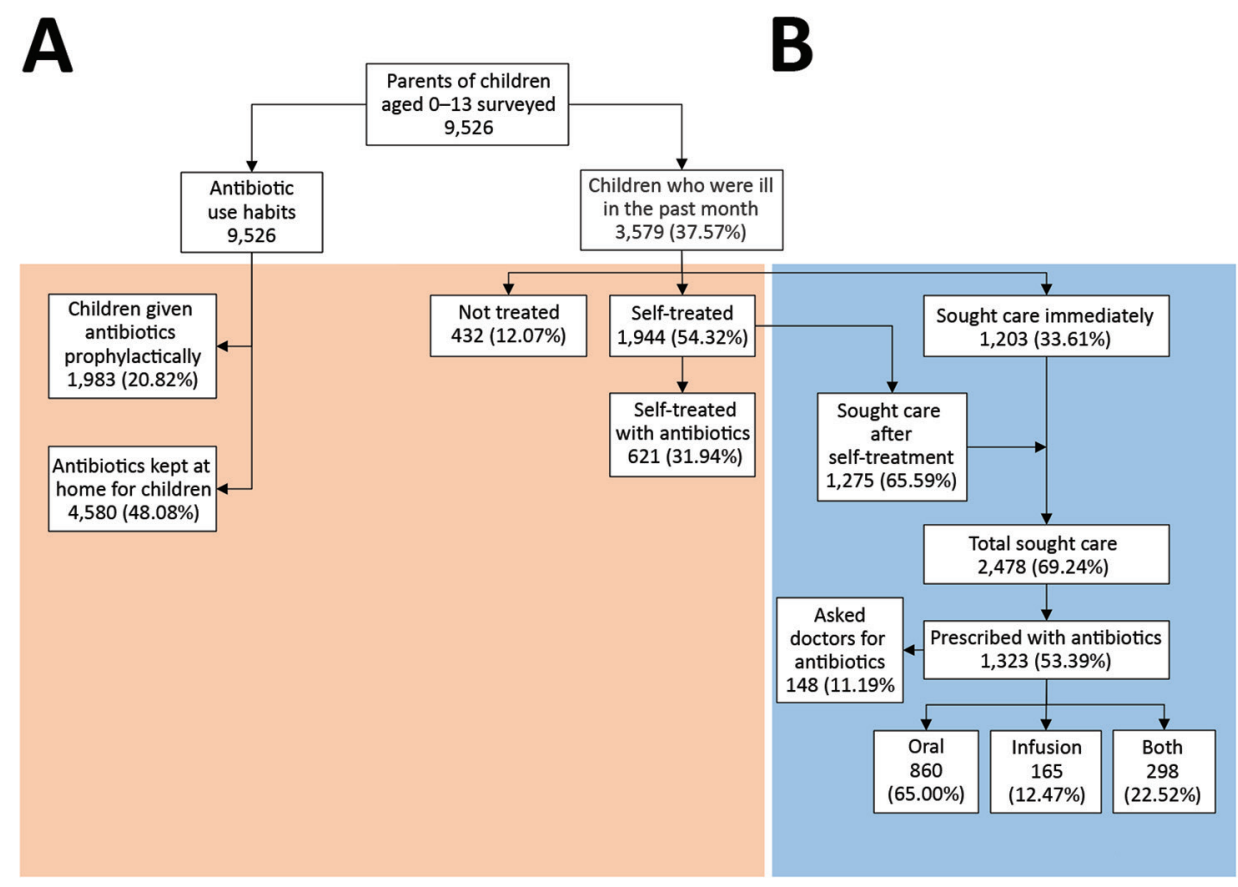

Figure 1. Use of antimicrobial drugs (antibiotics) for children by their parents $(N=9,526)$, China. Section A (left of dashed line) represents chronic antibiotic use in the previous year; section $B$ (right of dashed line) indicates antibiotic use for common childhood illnesses in the previous month. Orange shading indicates treatment within community or household, outside of a clinical setting. Blue shading indicates use within a clinical setting.

(Table). Most parents (55.2\%-78.5\%) were aware of the danger that overuse of antibiotics poses, yet confused antibiotics with inflammatory drugs or drugs effective for treating colds or alleviating symptoms (Figure 2). Among the respondents, 37.6\% (3,579/9,526) self-reported that their children experienced a minor illness within the previous month, with some overlap between symptoms; $82.1 \%(2,938 / 3,579)$ reported that it was a common cold with rhinitis, nasal congestion, or cough; $47.7 \%(1,707 / 3,579)$ sore throat; $31.0 \%$ $(1,108 / 3,579)$ fever; $12.5 \%(446 / 3,579)$ diarrhea; and $3.3 \%(119 / 3,579)$ otitis media. Among parents who reported to have self-treated sick children, 31.9\% $(621 / 1,944)$ reported medicating them with antibiotics from either a local pharmacy $(57.0 \%, 354 / 621)$ or personal stock $(33.3 \%, 207 / 621)$. Almost all household stock of antibiotics was reported to have come from leftover prescriptions $(63.1 \%, 2,891 / 4,580)$ or over-the-counter purchases $(35.3 \%, 1,619 / 4,580)$; community pharmacies accounted for $70 \%$ of antibiotics for self-medication of children.

Of 3,579 parents whose children were ill in the previous month, $69.2 \%(2,478 / 3,579)$ sought care for the child. Before seeing a doctor, $16.5 \%(410 / 2,478)$ of children had already been medicated with antibiotics at home; moreover, among them, $15.4 \%(63 / 410)$ of parents admitted to having then asked for more antibiotics at the facility. Among those for whom care was sought after parents medicated with antibiotics, $83.9 \%(344 / 410)$ were prescribed antibiotics, $17.2 \%$ $(59 / 344)$ of them because of parental demand. Data showed the success rate of obtaining antibiotic prescriptions for children was $79.6 \%$ (148/186); parental demand for antibiotics was more likely to occur in lower-level hospitals than tertiary hospitals and was associated with $\approx 4$-fold increase in prescribed

\begin{tabular}{|c|c|c|c|c|c|}
\hline \multirow[b]{2}{*}{ Study question } & \multirow{2}{*}{$\begin{array}{l}\text { Children brought } \\
\text { to medical facility, } \\
\mathrm{N}=2,478 \text {, no. }(\%)\end{array}$} & \multicolumn{4}{|c|}{ Children prescribed antibiotics, $n=1,323[53.39 \%]$} \\
\hline & & No. (\%) & OR & aOR $(95 \% \mathrm{Cl}) \dagger$ & $\mathrm{p}$ valuef \\
\hline \multicolumn{5}{|c|}{ What was the medical facility used when seeking care for your child? (urban/rural) } & 0.04 \\
\hline Tertiary hospital & $367(14.8)$ & $174(47.41)$ & Referent & Referent & \\
\hline Secondary/county hospital & $1,057(42.7)$ & $592(56.01)$ & $1.41(1.11-1.79)$ & $1.43(1.11-1.84)$ & \\
\hline $\begin{array}{l}\text { Community health centers/township } \\
\text { hospital }\end{array}$ & $719(29.0)$ & $373(51.88)$ & $1.20(0.93-1.54)$ & $1.17(0.90-1.53)$ & \\
\hline Private clinics/ village clinics & $335(13.5)$ & $184(54.93)$ & $1.35(1.00-1.82)$ & $1.21(0.89-1.65)$ & \\
\hline \multicolumn{5}{|c|}{ When seeking care at the medical facility, did you ask the doctor for antibiotics? } & $<0.0001$ \\
\hline No & $2,292(92.5)$ & $1,175(88.81)$ & Referent & Referent & \\
\hline Yes & $186(7.5)$ & $148(11.19)$ & $3.70(2.57-5.34)$ & $3.71(2.56-5.38)$ & \\
\hline
\end{tabular}




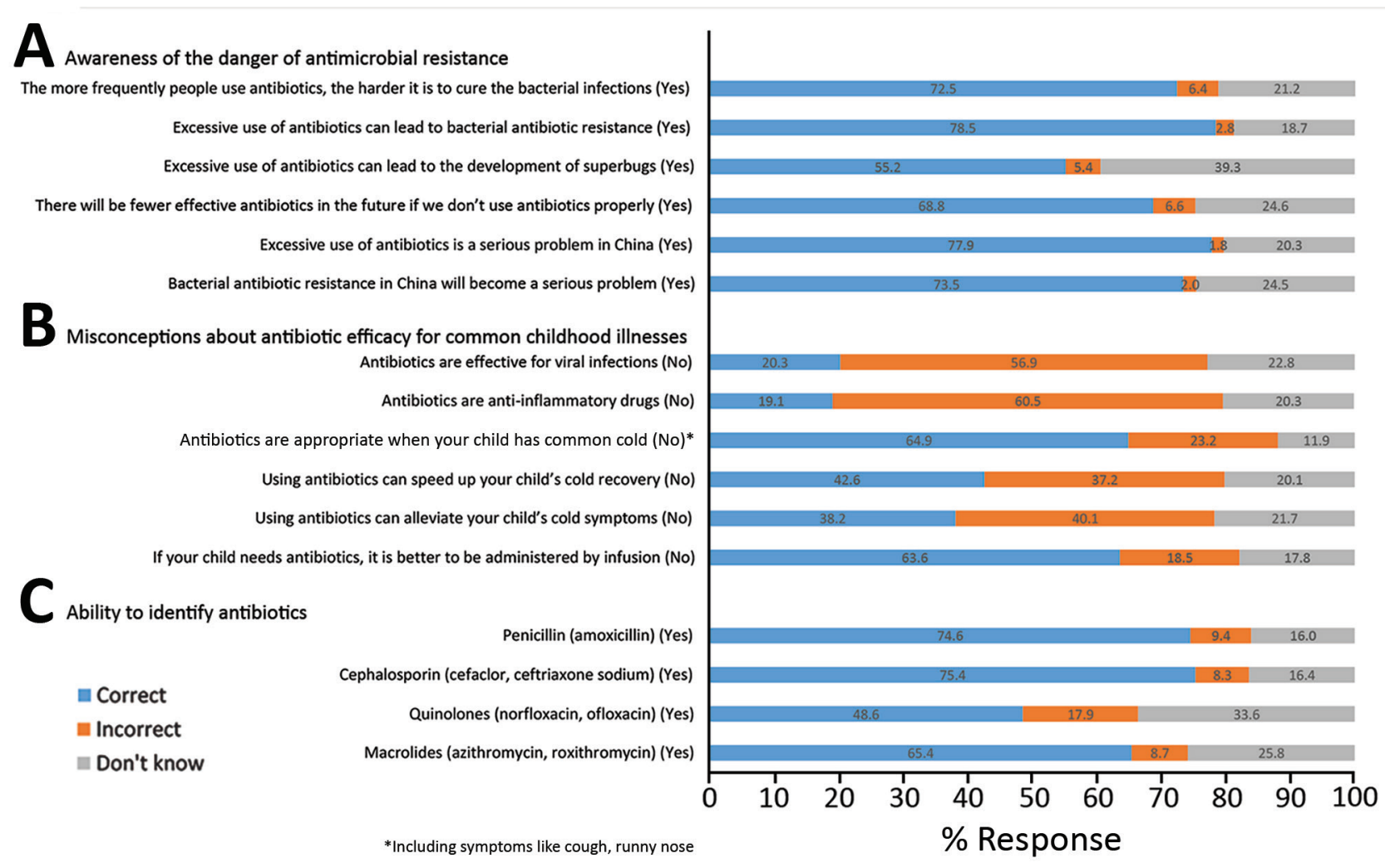

Figure 2. Answers to questions about antibiotics-related knowledge among parents in 3 representative provinces in China $(\mathrm{N}=9,526)$. A) Knowledge of risks for antimicrobial resistance; B) understanding of drug efficacy for common illnesses; C) drug recognition. Correct answers are shown in parentheses.

antibiotics (Appendix Table, https://wwwnc.cdc. gov/EID/article/26/8/19-0631-App1.pdf).

Overall, $53.4 \%(1,323 / 2,478)$ of children for whom care was sought were prescribed antibiotics. The most commonly prescribed antibiotic classes were penicillins, macrolides, and cephalosporins, either alone or in combination. Differences emerged in prescription rates by type of healthcare facility, ranging from $47.4 \%$ $(174 / 367)$ in tertiary hospitals to $56.0 \%(592 / 1,057)$ in county hospitals. More than $33 \%$ of children were administered intravenous antibiotics; about half of those infusions were combined with oral antibiotics.

Our data showed that, among the 3,579 children who had common minor childhood illnesses (mostly self-limiting) in the previous month, 621 were administered nonprescription antibiotics by their parents and 1,323 obtained a prescription, with 148 of those deemed inappropriate due to parental demand. We estimated that demand from parents contributed to $40 \%$ of antibiotic use on children for self-limiting illnesses. Although some doctors' prescriptions (supply-side) might be considered appropriate, all antibiotic demands and nonprescription uses from parents were inappropriate.

\section{Conclusions}

Overuse of medical care for self-limiting illnesses combined with a high prescription rate and a large population size drive high antibiotic consumption in China. We found that, of children for whom care was sought, $53.4 \%(1,323 / 2,478)$ received prescriptions for antibiotics; this proportion is at least twice as high as the official cap of outpatient prescriptions enforced in $2012(10,11)$. Unsupervised administration of antibiotics in children in the household, 3-10 times higher in China than in some countries in Europe (12), is a serious problem that has persisted for 15 years (13). Despite a 2004 ban on nonprescription sales of antimicrobial drugs $(10,11)$, participants in all sampled sites have been able to obtain them over the counter.

Enforcing existing stewardship policies is an important step to reduce inappropriate antibiotic use in the community (11), as is a multifaceted program that addresses drivers of inappropriate use from both sides of healthcare system. Such a program should provide parents with education about antibiotic efficacy and care for childhood illnesses (14), corresponding to children's developmental stages: prenatal care, vaccination, and kindergarten and 
primary school-age concerns. The program should also support healthcare providers by removing financial incentives to overprescribe medications and outpatient pediatric infusion services, enhancing clinical diagnostic capacity, and providing training on rational prescribing $(14,15)$. Finally, interventions to improve effective patient-physician interaction and communication should consider both sides of the healthcare system (14).

This study is limited by its cross-sectional design; it cannot be used to establish causal conclusions and is subject to recall bias. We limited questions about healthcare-seeking behaviors to the month before the survey and recruited a large sample to reduce the risk for bias. Because we estimated antibiotic consumption by a snapshot survey and not by prescriptions or use, the true magnitude of misuse in children may be underestimated. Common childhood illness cases reported in this study were diagnosed by parents; such diagnoses may or may not reflect true prevalence of the diseases, yet were a key determinant of parents' childcare behaviors that dictated healthcare decisions parents made for their children.

\section{Acknowledgments}

We thank the administrators and officials at the schools and health centers involved in disseminating this survey and coordinating the study for their assistance and support.

The study was funded by Zhejiang University Zijin Talent Program.

\section{About the Author}

Dr. Lin earned her PhD at the London School of Hygiene and Tropical Medicine, specializing in implementation science and evaluation, social epidemiology, and communication and behavior sciences. Her primary research interest includes the determinants of antibiotic use, epidemic preparedness, and development of multifaceted complex interventions to reduce inappropriate antibiotic use and resistance using cross-sectional, experimental, and longitudinal studies.

\section{References}

1. Tang Q, Song P, Li J, Kong F, Sun L, Xu L. Control of antibiotic resistance in China must not be delayed: the current state of resistance and policy suggestions for the government, medical facilities, and patients. Biosci Trends. 2016;10:1-6. https:/ / doi.org/10.5582/ bst.2016.01034
2. Heddini A, Cars O, Qiang S, Tomson G. Antibiotic resistance in China - a major future challenge. Lancet. 2009;373:30. https:/ / doi.org/10.1016/S0140-6736(08)61956-X

3. Quan-Cheng K, Jian-Guo W, Xiang-Hua L, Zhen-Zhen L. Inappropriate use of antibiotics in children in China. Lancet. 2016;387:1273-4. https:// doi.org/10.1016/ S0140-6736(16)30019-8

4. Wang CQ, Wang AM, Yu H, Xu HM, Jing CM, Deng JK, et al. Report of antimicrobial resistance surveillance program in Chinese children in 2016 [in Chinese]. Zhonghua Er Ke Za Zhi. 2018;56:29-33.

5. Chang J, Lv B, Zhu S, Yu J, Zhang Y, Ye D, et al. Non-prescription use of antibiotics among children in urban China: a cross-sectional survey of knowledge, attitudes, and practices. Expert Rev Anti Infect Ther. 2018;16:163-72. https://doi.org/10.1080/14787210.2018.1425616

6. Yu M, Zhao G, Stålsby Lundborg C, Zhu Y, Zhao Q, Xu B. Knowledge, attitudes, and practices of parents in rural China on the use of antibiotics in children: a cross-sectional study. BMC Infect Dis. 2014;14:112. https:/ / doi.org/10.1186/ 1471-2334-14-112

7. Cheng J, Chai J, Sun Y, Wang D. Antibiotics use for upper respiratory tract infections among children in rural Anhui: children's presentations, caregivers' management, and implications for public health policy. J Public Health Policy. 2019;40:236-52. https:/ / doi.org/10.1057/ s41271-019-00161-w

8. Sun C, Hu YJ, Wang X, Lu J, Lin L, Zhou X. Influence of leftover antibiotics on self-medication with antibiotics for children: a cross-sectional study from three Chinese provinces. BMJ Open. 2019;9:e033679. https:// doi.org/ 10.1136/bmjopen-2019-033679

9. National Bureau of Statistics of China. National data. 2017 [cited 2018 Sep]. http:/ / data.stats.gov.cn

10. Xiao Y, Li L. Legislation of clinical antibiotic use in China. Lancet Infect Dis. 2013;13:189-91. https:/ / doi.org/10.1016/ S1473-3099(13)70011-2

11. Wang L, Zhang X, Liang X, Bloom G. Addressing antimicrobial resistance in China: policy implementation in a complex context. Global Health. 2016;12:30. https:/ / doi.org/10.1186/s12992-016-0167-7

12. Morgan DJ, Okeke IN, Laxminarayan R, Perencevich EN, Weisenberg S. Non-prescription antimicrobial use worldwide: a systematic review. Lancet Infect Dis. 2011;11:692701. https:/ / doi.org/10.1016/S1473-3099(11)70054-8

13. Bi P, Tong $S$, Parton KA. Family self-medication and antibiotics abuse for children and juveniles in a Chinese city. Soc Sci Med. 2000;50:1445-50. https:/ / doi.org/10.1016/ S0277-9536(99)00304-4

14. Wei X, Zhang Z, Hicks JP, Walley JD, King R, Newell JN, et al. Long-term outcomes of an educational intervention to reduce antibiotic prescribing for childhood upper respiratory tract infections in rural China: follow-up of a cluster-randomised controlled trial. PLoS Med. 2019; 16:e1002733. https://doi.org/10.1371/journal.pmed.1002733

15. Ding G, Vinturache A, Lu M. Addressing inappropriate antibiotic prescribing in China. CMAJ. 2019;191:E149-50. https://doi.org/10.1503/cmaj.181417

Address for correspondence: Xudong Zhou, Institute of Social and Family Medicine, School of Medicine, Zhejiang University, 866 Yuhangtang Rd, Hangzhou, Zhejiang, 310058, China; email: zhouxudong@zju.edu.cn 